\title{
REGION BASED MEDICAL IMAGE REGISTRATION APPROACH USING A MODIFIED ARTIFICIAL IMMUNE SYSTEM
}

\author{
Osama A. Omer ${ }^{1}$ and Mohamed Abdel-Nasser ${ }^{2}$ \\ Electrical Engineering Department, Aswan University \\ Aswan 81542, Egypt \\ 1omer.osama@gmail.com, 2egnaser@gmail.com
}

(Received September 2, 2012 Accepted November 7, 2012)

\begin{abstract}
A high accuracy medical image registration is needed to achieve an efficient medical diagnosis and computer aided surgeries, so that a novel region based medical image registration approach is presented in this paper. This approach consists of four sequential steps, image segmentation, point correspondence using a modified artificial immune system, point selection and finally LMS technique is used to estimate the warp parameters. The proposed approach provides a high accuracy and it doesn't require any additional treatment or feature extraction as some other methods do. To demonstrate the effectiveness of the proposed approach it tested for many image pairs and it is compared with many registration algorithms such aspoint-based image registration using an artificial immune system (AIS),ICP and RANSAC algorithms.
\end{abstract}

Keywords: Segmentation, AIS, correspondence, registration.

\section{Introduction}

In the recently years, image registration is used widely in medical diagnostic and medical surgeries, there are a lot of registration methods, these methods can be classified into two categories; intensity-based and feature-based. The registration process consists of two basic steps. The first is to determine the similarity (fitness) function, the choice of an image similarity measure depends on the modality of the images to be registered, common examples of image similarity measures include crosscorrelation [1], mutual information, sum of squared intensity differences. The second step is to solve the registration model, there are many common minimization strategies have been applied to image registration problems, such as exhaustive search, gradient descent [2], simulated annealing and genetic algorithm (GA) [3].

For its accuracy, GA algorithms have special interest in this context. Janko et al. [4] presented a successful application of GA to the registration of uncelebrated optimal images. F. L. Seixaset. et. al. [5] proposed image registration using genetic algorithm, in this work the point matching problem was addressed employing a method based on nearest neighbour however, this paper not evaluating the GA considering a real-world application. K. K. Delibasis et. al [6] proposed automatic point correspondence using an artificial immune system optimization technique for medical image registration, in this paper a modified mutation formula based on an uniform distribution was used. F. Ye et. al [7] proposed two step image registration by artificial immune system and chamfer matching, in this paper the artificial immune system has been used to find an 
initial transformation where the edge distance used as a fitness function then an area based method has been used to refine the transformation estimation.

Traditional optimization methods are easy to fail being trapped in one of the local minima so a global optimization technique is often required, the artificial immune systems are used for function optimization, the clonal selection and affinity mutation principles are used to explain how the immune systems perform the optimization process. There are many artificial immune systems were published in the context. An immune algorithm, named CLONALG, was developed to perform pattern recognition and optimization. De Castro and J. Timmis proposed opti-aiNet for multimodal function optimization [8]. This algorithm is used in our registration approach. The mutation formula proposed at [8] was:

$$
c^{-}=c+\alpha \cdot N(0,1), \alpha=(1 / \beta) \cdot \exp \left(-f^{*}\right)
$$

Where $\mathrm{c}^{-}$is the mutated component, $\mathrm{c}$ is the old component, $\mathrm{N}(0,1)$ is a random variable with Gaussian distribution with zero mean and unity standard deviation, $\alpha$ is the mutation ratio, $\mathrm{f}^{*}$ is the fitness for an individual (solution) normalized between the interval $[0,1], \beta$ is a parameter that controls the decay of the inverse exponential function. In [6] instead of a Gaussian random variable an uniform random variable is used, however we study the distribution of warping parameter as will be shown in the next section, we found that the parameter distribution is more Gaussian than uniform.

On the other hand, region-based optical flow estimation [9] was proposed by C. Fuh and P. Maragos, in this paper used the centroid of each segmented region in the reference and input image as features to estimate the correspondence. Even if the region-based optical flow estimation is suitable for objects motion, the main problem in this algorithm is that the centroid, as a feature, is not immune against noise and a translation model was used which is a simple model. O. A. Omer proposed region based optical flow estimation [10], in this algorithm the registration model is assumed to be translational which may be not proper to describe the complete motion in the medical images.

In this paper, we proposed a generalization for an initial work presented in [11], where a translation model was used, instead in this paper an affine model is used. To overcome the problems presented in [6] and [9-10], a region-based artificial immune system is proposed. To do that, the main contributions of this paper are; 1) study the distribution of the motion parameters, 2) modifying the artificial immune system and mutation formula proposed at [8] to be suitable for image registration then apply it to segmented regions, 3) and generalize the motion model to be suitable for affine motions.

To confirm the effectiveness of the proposed approach, it is compared with the point-based image registration using an artificial immune system approach [6], the iterative closest point (ICP) algorithm and the random sample consensus (RANSAC) algorithm.

\section{The distribution of the affine Warp parameters}

The distribution of the warp parameters has been studied between many pairs of images [11]. In this study; the first image of the car sequence is used as a reference image and the reset 63 images of the car sequence as input images, the additive lucaskanade (LK) registration algorithm is used to determine the six affine parameters (p1- 
p6), the number of iteration used is 200 iterations. The distribution of the six affine parameters are shown in Figure 1, the scaling parameter along $\mathrm{x}$ - axis $\mathrm{p} 1$ is shown in (a), p4 the scaling parameter a long $y$ - axis is shown in (b), the shearing parameter along $\mathrm{x}$ - axis $\mathrm{p} 2$ is shown in (c), the shearing parameter along $\mathrm{y}$ - axis $\mathrm{p} 3$ is shown in (d), p5 is the translation along $\mathrm{x}$-axis shown in (e), finally the translation along $\mathrm{y}$-axis p6 is shown in (f). It is easy to notice that the parameters distribution is not uniform as introduced in [6] and it can be considered as Gaussian distribution. Based on this study in the mutation part we will modify Eq. (1) to be suitable for image registration process.

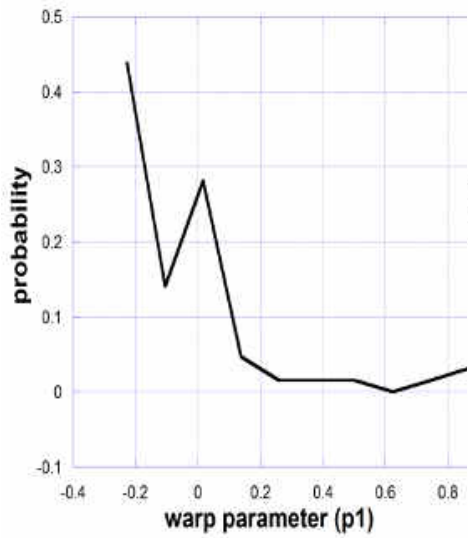

(a)

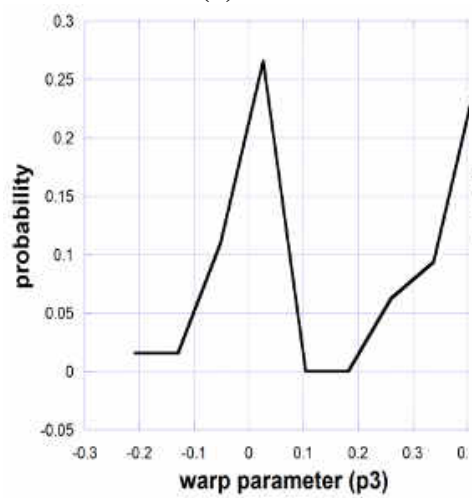

(d)

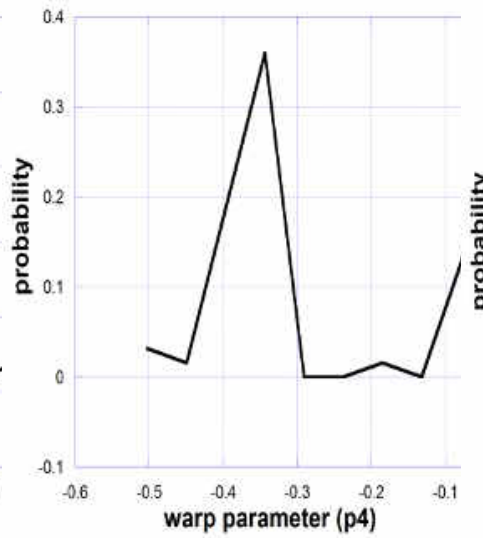

(b)

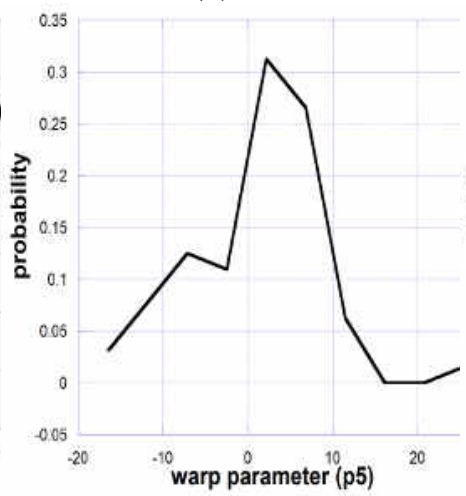

(e)

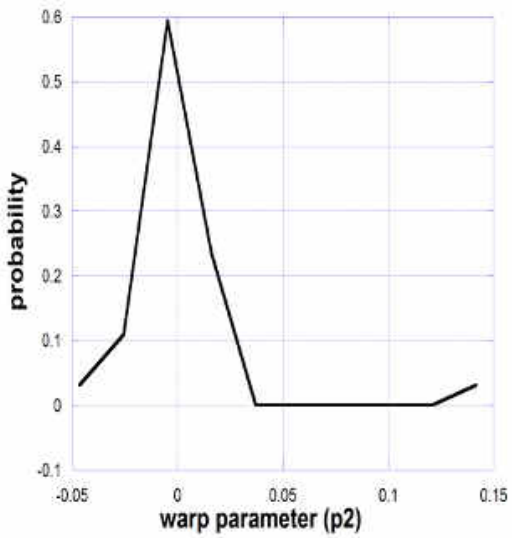

(c)

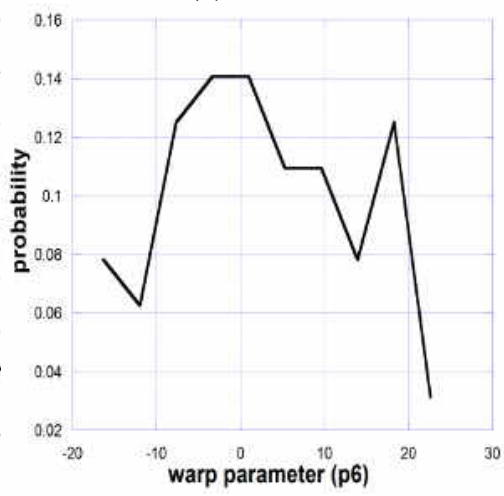

(f)

Figure 1 The affine parameters distribution, (a) p1, (b) p4, (c) p2, (d) p3, (e) p5, (f) p6.

\section{The proposed approach}

The proposed approach consists of four sequential steps as shown in Figure 2 below. The first step is to segment the reference image using watershed segmentation method and suppress the small regions. The second step is to find a number of correspondence regions between the reference and the input images by correspondence algorithm based on a modified opti-aiNet algorithm. The third is the selection step. Finally, the least squares technique used to determine the transformation which is used to align the reference and input images. These steps are described in details as follows: 
3.1 The segmentation the reference image

The regions are extracted using the watershed segmentation method, here the segmentation process is performed only at the reference image then these regions are used as an input for the next step.

\subsection{The modification of the artificial immune system}

The modification on opti-aiNet algorithm is that, the suppression and regeneration stages are canceled because the mutation of each cell was limited to its region.

\subsection{The registration model}

The affine transformation is used as a registration model [12], this model has six degree of freedom (6 DOF), and this model consists of six parameters (p1-p6) as shown in (2)

$$
\left[\begin{array}{l}
x^{-} \\
y^{-}
\end{array}\right]=\left[\begin{array}{ll}
p 1 & p 3 \\
p 2 & p 4
\end{array}\right]\left[\begin{array}{l}
x \\
y
\end{array}\right]+\left[\begin{array}{l}
p 5 \\
p 6
\end{array}\right]
$$

\subsection{The population arrangement}

The population is initialized in the search range with $\mathrm{N}$ row (cell). The arrangement of the population is described in Figure 3, where each row represents a cell. Each cell consists of eight components $(\mathrm{x}, \mathrm{y}, \mathrm{p} 1, \mathrm{p} 2, \mathrm{p} 3, \mathrm{p} 4, \mathrm{p} 5, \mathrm{p} 6)$, where $\mathrm{x}$ and $y$ is the location of a point which randomly token from each region in the reference image, (p1-p6) the affine parameters, $f$ is the fitness of the population row and ind is the index of the population row .

\subsection{The fitness function}

The fitness is calculated for each cell from the population, the squared of normalized cross correlation has been used as a fitness function, its values lie between the interval $[0,1]$ so it can be used directly. The fitness function formula is described below in Eq. (3) where a1 is the region which contains the point of population cell, the coordinates of a1 is warped using it's parameters (p1-p6) to give a2 which is the correspondence region that includes the correspondence pixel in the input image, $\mathrm{m} 1$ and $\mathrm{m} 2$ are the mean of $\mathrm{a} 1$ and $\mathrm{a} 2$ respectively.

$$
\text { fitness }=\frac{\left[\sum_{x, y}(a 1(x, y)-m 1)(a 2(x, y)-m 2)\right]^{2}}{\sum_{x, y}(a 1(x, y)-m 1)^{2} \sum_{x, y}(a 2(x, y)-m 2)^{2}}
$$

\subsection{The clone mutation}

In the mutation stage, a number of $\mathrm{n}_{\mathrm{c}}$ clones are created for each cell then these clones are mutated, the clone which has the highest fitness is selected and if this clone has fitness bigger than the original population cell fitness then it replaced by this clone. In this paper a modified mutation formula is proposed where Eq. (1) is adjusted to be suitable for image registration purpose. Each cell component is mutated according to Eq. (4). 


$$
\begin{gathered}
c^{-}=c+\alpha \cdot N(0,1) \\
\alpha=\exp \left(-f^{*}\right) \cdot\left(S_{\text {max }}-S_{\text {min }}\right)
\end{gathered}
$$

Where $\mathrm{c}^{-}$is the mutated component, $\mathrm{c}$ is the old value of the components, $\mathrm{f}^{*}$ is the normalized fitness function, $S_{\max }$ is maximum value of the parameter, $S_{\min }$ is minimum value of the parameter. Each cell component is mutated so that the location of the new clone $(\mathrm{x}, \mathrm{y})$ lies in the coordinates of the region which contains this point and any clone exceed $S_{\max }$ or $S_{\min }$ is refused.

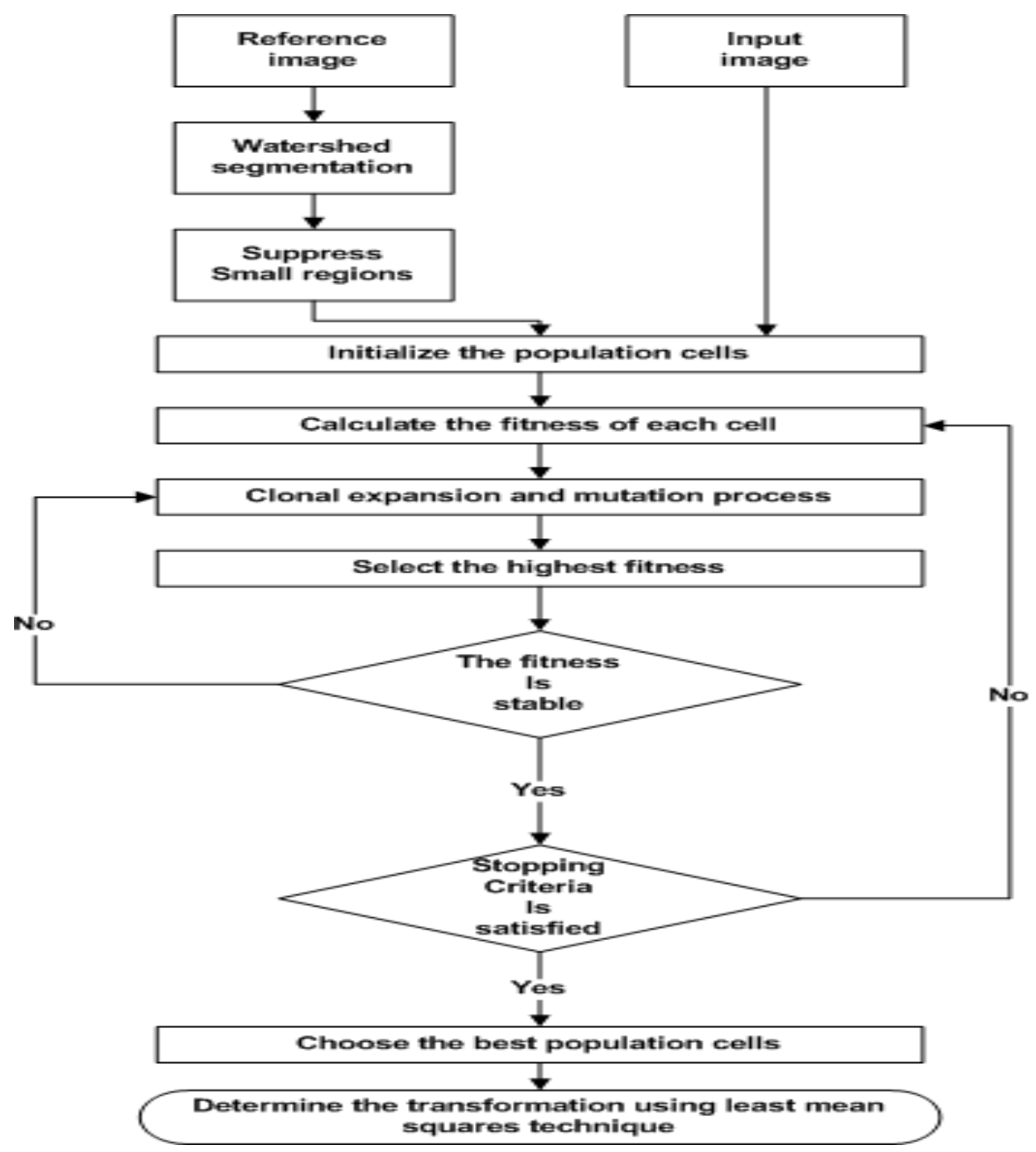

Figure 2 The proposed registration approach 


$$
\operatorname{pop}=\left[\begin{array}{cccccccccc}
x 1 & y 1 & p 1 & p 2 & p 3 & p 4 & p 5 & p 6 & f_{1} & \text { ind }_{1} \\
x 2 & y 2 & p 1 & p 2 & p 3 & p 4 & p 5 & p 6 & f_{2} & \text { ind }_{2} \\
\cdot & \cdot & \cdot & \cdot & \cdot & \cdot & \cdot & \cdot & \cdot & \cdot \\
\cdot & \cdot & \cdot & \cdot & . & . & . & . & . & \cdot \\
x_{n} & y_{n} & p 1 & p 2 & p 3 & p 4 & p 5 & p 6 & f_{n} & \text { ind }_{n}
\end{array}\right]
$$

Figure 3 population arrangement

\subsection{Clonal expansion and the mutation reparation}

The process of clonal expansion and the mutation are repeated until the average fitness is stabilized and dropped below a predefined threshold. All the previous processes are repeated until the predefined number of iteration is reached; here the correspondence step is completed.

\subsection{Selection}

The population cells which have fitness higher than a predefined value has been chosen and the other cells are removed from the population.

\subsection{Warp parameters estimation}

The least mean squares LMS technique [13] is used to estimate the transformation parameters between the reference and input images, then these parameters is used to align the input image to the reference image.

\section{Simulation results and discussion}

\subsection{Data set description}

In this paper, three experiments are deducted on three different medical images including brain image, retinal image and hand image to demonstrate the effectiveness of the proposed approach; these images have a size $160 \times 160,137 \times 168$ and $128 \times$ 128 respectively. The initial number of cells in the population $\mathrm{N}$ used is 30 , we used ten clones $\left(\mathrm{n}_{\mathrm{c}}=10\right)$ for each cell, the average fitness is stabilized when it drops below 0.001 , we used 100 iteration to perform this process, and finally all cells which have a fitness less than 0.97 are removed from the population. The watershed segmentation is used to segment the reference image with 100 segmentation threshold then all regions that contains less than 40 points are suppressed. The watershed segmentation based on rain-falling simulation [14] is used in this paper. This approach was implemented in MATLAB language; all the previous parameters are tuned experimentally.

\subsection{Comparative results}

In this section, we test the proposed approach using three different medical images, brain, retinal and hand. The proposed approach accuracy is compared with point-based image registration using an artificial immune system approach proposed at [6] with the modified mutation formula (Eq. 4). Also, the proposed approach has been compared with two state-of-the-art point-based registration approaches. The first approach based on the ICP algorithm [15-16]. The second approach based on the RANSAC algorithm 
[17-18]. Both approaches need a feature extraction step to perform the registration process; in this paper we used the well-known Harris corners [19] to extract the control points from the reference and input images. In Table 1, the main differences among the proposed approach, ICP and RANSAC algorithms has been summarized for different viewpoints.

Table 1 Comparison

\begin{tabular}{|l|c|c|c|}
\hline Viewpoints & $\begin{array}{c}\text { The proposed } \\
\text { approach }\end{array}$ & ICP & RANSAC \\
\hline $\begin{array}{l}\text { Feature } \\
\text { detector }\end{array}$ & Not needed & $\begin{array}{c}\text { Mandatory } \\
\text { step }\end{array}$ & $\begin{array}{c}\text { Mandatory } \\
\text { step }\end{array}$ \\
\hline $\begin{array}{l}\text { Warp parameters } \\
\text { search range }\end{array}$ & Pre-Set & Not controlled & Not controlled \\
\hline Performance & Excellent & $\begin{array}{c}\text { Depend on } \\
\text { The feature } \\
\text { extraction step }\end{array}$ & $\begin{array}{c}\text { Depend on } \\
\text { The feature } \\
\text { extraction step }\end{array}$ \\
\hline $\begin{array}{l}\text { Max. no. of } \\
\text { iteration }\end{array}$ & 100 & 104 & 1001 \\
\hline
\end{tabular}

$$
\operatorname{PSNR}=\frac{f_{\text {max }}^{2}}{E\left[\left(f_{\text {ref }}-f_{\text {reg }}\right)^{2}\right]}
$$

The peak signal to noise ratio (PSNR) in $\mathrm{dB}$ is used to compare the performance of the proposed approach against ICP and RANSAC approaches. PSNR is defined as:

Where $f_{\max }$ is the maximum intensity of the image, $E[$.$] is the ensemble average, f_{\text {ref }}$ is the reference image intensity and $f_{\text {reg }}$ is the registered image intensity. The PSNR is calculated only for the overlapped part between the reference and input images.

The visual results of the comparison between the performances of the three approaches with the medical images (brain, retinal and hand images) are shown in Figure4, Figure 5 and Figure 6 respectively. Table 2 shows that the proposed approach has the best performance compared to Ref. [6], ICP and RANSAC approaches because it achieves the highest PSNR. The results show that the proposed algorithm is very

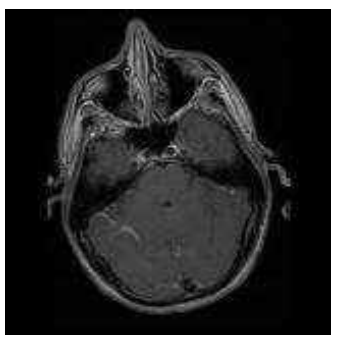

(a)

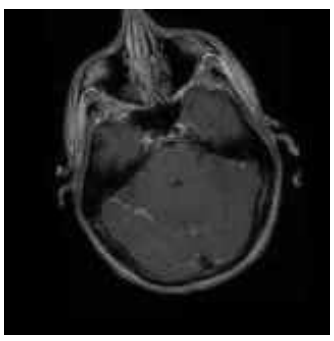

(b)

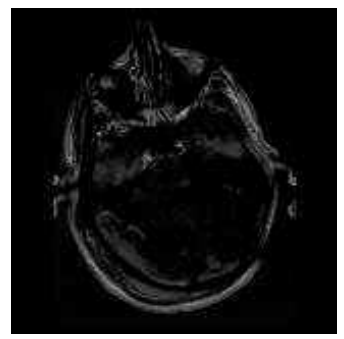

(c)

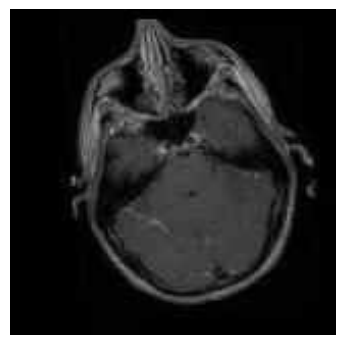

(d) 


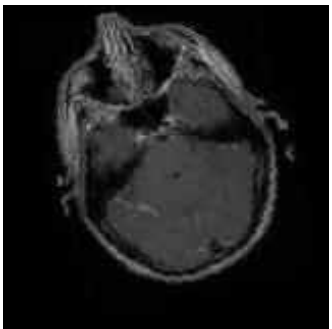

(e)

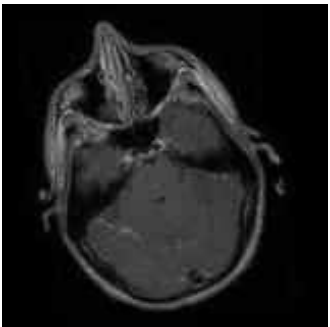

(f)

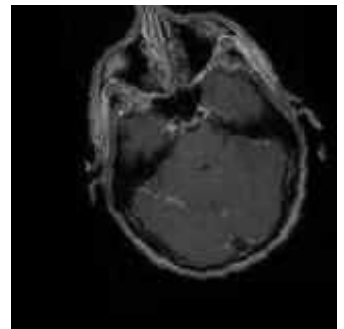

(g)

Figure 4 (a) The reference image, (b) The input image, (c) The difference between the reference and input image, (d-g) The registered image in the case of the proposed approach, AIS [6], ICP and RANSAC respectively.

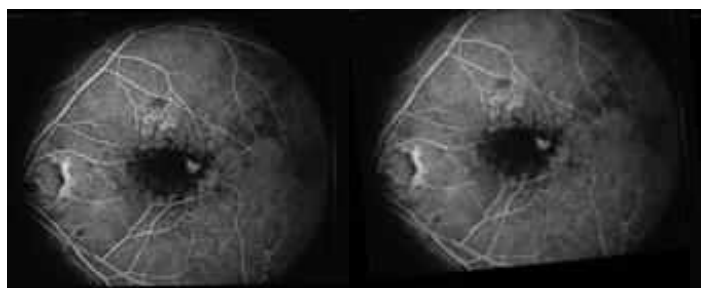

(a)

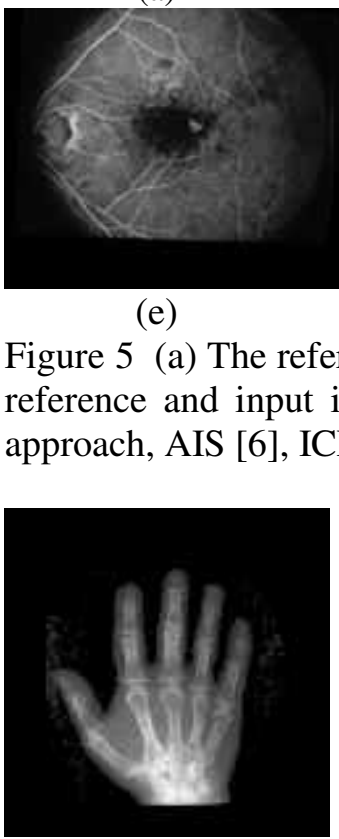

(a)

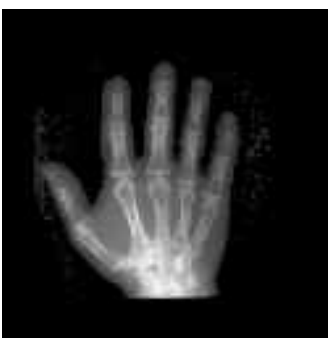

(b)

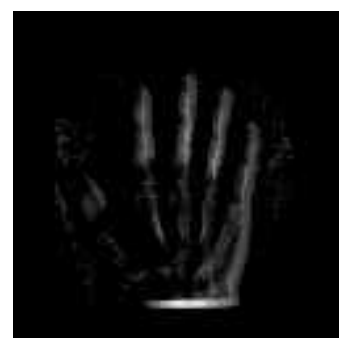

(c)

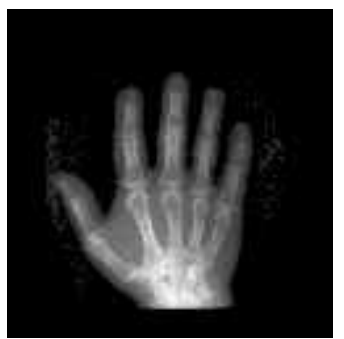

(d) 


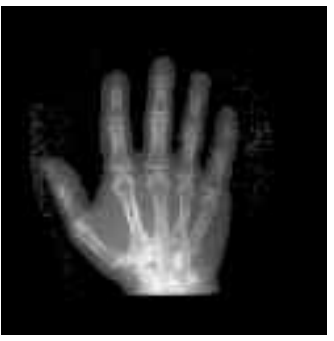

(e)

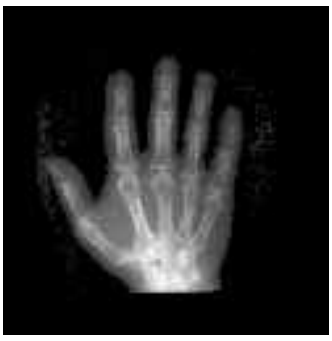

(f)

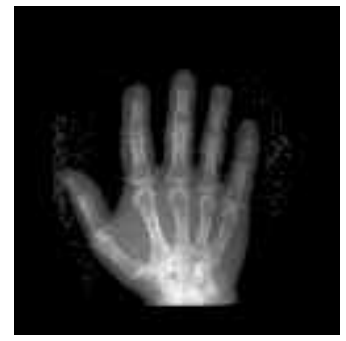

(g)

Figure 6 (a) The reference image, (b) The input image, (c) The difference between the reference and input image, $(\mathrm{d}-\mathrm{g})$ The registered image in the case of the proposed approach, AIS [6], ICP and RANSAC respectively

Suitable to register medical image, on the other hand the ICP and RANSAC approaches may fail to register the medical images as it clear when the RANSAC used with the retinal image as it shown in Figure $5(\mathrm{~g})$ and Table 2.

Table 2 PSNR comparison

\begin{tabular}{|l|c|c|c|c|}
\hline Image pairs & The proposed & AIS [6] & ICP & RANSAC \\
\hline Brain image & 37.4172 & 37.1441 & 35.2053 & 34.3397 \\
\hline Retinal image & 35.8691 & 34.5416 & 31.2473 & 26.8285 \\
\hline Hand image & 56.5865 & 41.4221 & 56.5496 & 56.5496 \\
\hline
\end{tabular}

\section{Conclusion}

In this paper, a new approach for medical image registration has been presented, this approach consists of four sequential steps, image segmentation and small region suppression, point correspondence using a modified artificial immune system, selection and finally the LMS minimization technique is used to estimate the warp parameters. The simulation results which based on the affine registration model show that the proposed approach outperforms the point-based image registration using an artificial immune system, ICP and RANSAC algorithms. The results show that also the RANSAC and ICP may be failed to register the medical images. The future work will focused on study the performance of the proposed approach with multimodal medical images.

\section{References}

[1] D. Deguchi, K. Mori, M. Feuerstein, T. Kitasaka, C. R. Maurer , Y. Suenaga , H. Takabatake, M. Mori, and H. Natori "Selective image similarity measure for bronchoscope tracking based on image registration", Medical Image Analysis, vol. 13, No. 4, pp. 621-633.,2009.

[2] S. Baker and I. Matthews, 'Lucas-Kanade 20 years on: a unifying framework: part 1," tech. report CMU-RI-TR-02-16, Robotics Institute, Carnegie Mellon University, pp.1-10, July, 2002.

[3] M. Johansson, "Image Registration with Simulated Annealing and Genetic Algorithms", MSc Thesis, pp. 15-36, Stockholm, Sweden 2006.

[4] Z. Jankó, D. Chetverikov and A. Ekárt," Using a genetic algorithm to register an uncalibrated image pair to a 3D surface model", Engineering Applications of Artificial Intelligence, vol. 19,No. 3, pp. 269-276, 2006. 
[5] F. L. Seixas, L. S. Ochi, A. Conci and D. C. Saade ," Image Registration Using Genetic Algorithms”, GECCO’08, pp. 1145-1146, July 12-16, Atlanta, Georgia, USA, 2008.

[6] K. K. Delibasis, P. A. Asvestas and G. K. Matsopoulos,“ "Automatic point correspondence using an artificial immune system optimization technique for medical image registration", Computerized Medical Imaging and Graphics 35, pp.31-41, 2011.

[7] Famao Ye, ShaopingXu, and YuhongXiong, " Two-step image registration by artificial immune system and chamfer matching", Chinese Optics Letters, Vol. 6, Issue 9, pp. 651-653, 2008.

[8] L. N. De Castro and J. Timmis, "An Artificial Immune Network for Multimodal Function Optimization", Congress on Evolutionary Computation (CEC'02), vol. 1, pp. 699-674, 2002.

[9] C. Fuh and P. Maragos , "Region-based optical flow estimation," in Proc. of Computer Vision and Pattern Recognition (CVPR), pp. 130 - 135 ,June 1989.

[10] O. A. Omer, "Region-based Horn-Schunck Optical Flow Estimation," in proceeding of Japan-Egypt Conference on Electronics, Communications and Computers (JEC-ECC), pp. 73-78, March 6-9, 2012.

[11] Mohamed Abdel-Nasser and Osama A. Omer, "Region based image registration approach using an artificial immune system”, NRSC, 263-270, Cairo, April 2012.

[12] Isaac N. Bankman, "Handbook of medical imaging", pp. 421-612, Academic Press, 2000.

[13] Lecture notes on himage registeration, http://www.cs.rpi.edu/academics/courses/spring04/imagereg, 2011.

[14] P. De Smet and D. De Vleschauwer, "Performance and scalability of highly optimized rain falling watershed algorithm," in Proc. of the International Conference on Imaging Science, Systems and Technology, CISST98, pp. 266-273, Las Vegas, NV, USA, July 1998.

[15] P. J. Besl and N. D. McKay, "A method for registration of 3-D shapes" IEEE Trans. Patt. Anal. Mach. Intell. Vol. 14, No. 2, pp. 239-256,1992.

[16] A. A. Goshtasby, "2-D and 3-D Image Registration for medical, remote sensing and industrial applications "chapter3, pp. 43-61 ,John Wiley \& Sons, Inc. ,2005

[17] M.A. Fischler and R.C. Bolles. Random sample consensus: A paradigm for model fitting with applications to image analysis and automated cartography. Communicationsof the ACM, 24(6):381-395, 1981.

[18] T. Colleu, J. Shen, B.J. Matuszewski, L.Shark and Claude Cariou, "Feature-Based Deformable Image Registration with RANSAC Based Search Correspondence", AECRIS'06 - Atlantic Europe Conference on Remote Imaging and Spectroscopy, pp. 57-64, Preston, UK, September, 2006.

[19] Harris, C. and M. Stephens, "A combined corner and edge detector", AlveyVision Conference, 147-151, 1988. 
طريقة محاذه للصور الطبيه معتمده على المناطق باستخدام نظام مناعه اصطناعي معدل إجراء التشخيص الطبي والعمليات الجراحيه بمساعدة الحاسب بكفاءه عاليه ينطلب محاذاه للصور الطبيه بدقه عاليه؛ لذلك نقدم في هذا البحث طريقه جديده لمحاذاة الصور الطبيه معتده على مناطق الصوره باستخدام نظام مناعه اصطناعي معدل. هذه الطريقه تتكون من اربعة مراحل منتاليه؛ تقطيع الصوره؛ ايجاد النقاط

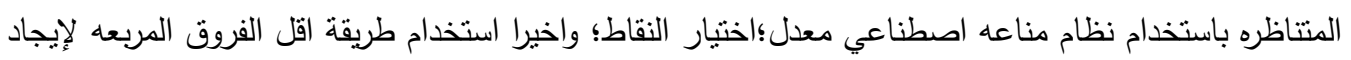
معاملات الحركة. هذه الطريقه المقترحه ذات دقه عاليه ولا تتطلب اي تعديلات اضافيه او استخلاص لخواص الصوره كما تتطلب بعض الطرق الاخرى. للالاله على فاعلية هذه الطريقة المقترحه فقد تم اختبارها على عدة

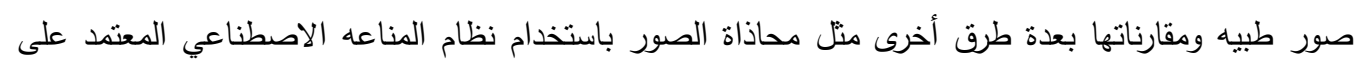
النقاط؛ طريقه معتمده على النقطه الأقرب بالتكرار وطريقه معتمدة على الإجماع على العينة العشوائيه. 